\title{
Study Buddy: An Emotionally Intelligent Tutoring System
}

\author{
Drishti Kanjilal \\ VIT University \\ BK 371 Sector 2,Salt Lake City \\ Kolkata, West Bengal,India
}

\begin{abstract}
With the advent of technology, online education has come to the forefront with millions of online courses attracting users from all over the world. To aid and abet the rising popularity of these courses, emotionally intelligent computer software is required that can deal with the varied needs of the user as opposed to a general course for everyone. A system is proposed that can effectively gauge the learner's capabilities, cognitive states and frame of mind by analyzing their expressions, learning speed and other factors. Such a system will radically change the world of online and distance education.
\end{abstract}

\section{General Terms}

Artificial intelligence extended to detect emotions and help improve online learning in MOOCs.

\section{Keywords}

Artificial emotional intelligence; MOOCs; online tutoring; AI; tutoring system

\section{INTRODUCTION}

In this digital age, online education has come to the forefront with millions of people, both students and professionals using these courses to extend their knowledge or clarify concepts. Thus, MOOCS (Massive Open Online Course) are rapidly increasing in popularity and content, with professors from global top universities teaching millions of users from the entire world. However, the downside to these courses is that no individual attention is given to students and the content of the course remains the same for all users. It is essential to realize that all users are not alike; some might be school students while others might be professionals. Each user will have different learning rates, abilities and problem areas. The current systems are unable to deal with this variety of users, thus making a standard set of videos or materials for the average user. It is time to introduce emotionally intelligent computer programs which can assess the students' abilities, cognitive states and moods and cater to their specific needs [2].

Computers are highly efficient and fast and perform human tasks better. The only barrier between machines and humans is the ability to express and understand emotions. Artificial emotional intelligence or affective computing is working towards bridging this gap [1].The developments in this field will improve the education system immensely, especially with the rising popularity of MOOCs, which are designed for the average user and cannot satisfy each and every individual. By creating software that incorporates emotional intelligence in MOOCs, the software can determine how well the student grasps concepts and alter the content of the video or study materials. This will lead to more variations in video recordings and text for slow, average and fast learners thus, ensuring that the user is not over or underwhelmed.

This paper discusses the existing systems of artificially intelligent computers that exhibit some degree of emotional understanding and proposes a new emotionally intelligent tutoring system with emphasis on its merits.

\section{EXISTING SYSTEM}

The following systems exhibit emotional intelligence to various degrees. Some have been used in tutoring systems.

\subsection{Auto Tutor}

It is an intelligent system that emulates human tutoring by speaking in the natural language with basic gestures and expressions. It holds lengthy dialogues with the user during the course, which is analyzed to find out the cognitive state of the learner i.e. how much the user has understood. It then dynamically adapts to what the learner knows. Recent advancements have made adaptations according to emotions of the user in addition to what the user is perceived to have understood. It covers a certain number of topics like Newtonian physics, computer literacy and critical thinking. [3],[4]. This system is a stepping stone for highly advanced emotionally intelligent systems that can effectively understand emotions and learning capacities of the user.

\subsection{Affectiva}

It is facial recognition software that analyzes expressions and physiological responses to detect human feelings [2]. Expressions and responses can be linked with different types of emotions or feelings of people. The concept behind this software is comparing the expression and gestures of a user with an existing database of similar effects and the emotions associated with those expressions and gestures [5].

\subsection{Beyond Verbal}

Beyond Verbal Communication, Ltd. created Beyond Verbal, which is a patented technology that gets a person's full set of emotions and character traits by analyzing their voice in real time. It uses voice recognition software to analyze the pitch, volume, timing, pause and energy of the human voice as the users speak and decodes the emotion they are feeling [7],[8].

\subsection{Facial recognition software by NCSU}

Researches at North Carolina State University have developed a sort of "feeling computer" that has facial recognition software which can identify when a student is feeling frustrated, bored, etc., and respond accordingly. This was done by filming tutoring sessions of various students. The software analyzed the facial expressions and reported the emotions that the students were feeling accurately i.e. the computer could tell when students were struggling, felt challenged or were bored.[2] The above system can be used to 
judge the students cognitive and physiological states as it can understand to a certain extent what the student has understood and whether he or she finds the tutorial interesting. This can be further expanded to modulate the content of the tutorial based on student requirements and wants. This idea is projected in the paper.

All these advancements are close to bridging the gap between computes and humans; however there is still a lot to achieve. Human emotions are complex and are not completely understood by us let alone machines. So far, intelligent machines can recognize and remember data patterns from the physiological symptoms of emotions .For example, Furrowed brow means worried; high heart rate means excited; loud voice means angry [2]. Some emotions are not detectable and some symptoms can be correlated with various emotions like fast heartbeat can indicate fear or excitement, a frown can mean worry or concentration. Each human is unique and has a different way of expressing emotions [10].

Based on the existing systems, the following system is proposed that incorporates various aspects of the existing ones.

\section{PROPOSED SYSTEM}

The system will ask each student to select some options on enrollment such as name, age, ethnicity, level of education, etc. The software will start the course in slow, average or advanced mode depending on the mentioned factors like age and educational qualification. Throughout, the system shall observe the users' facial expressions while learning the material, voice tone during speaking answers and the time taken to complete each section with quizzes [10]. Based on these factors, the content will altered to suit the user's specific requirements as detailed below. The prerequisite of such a system will be availability of the course in different modes, with each mode having an interactive or theoretical option.

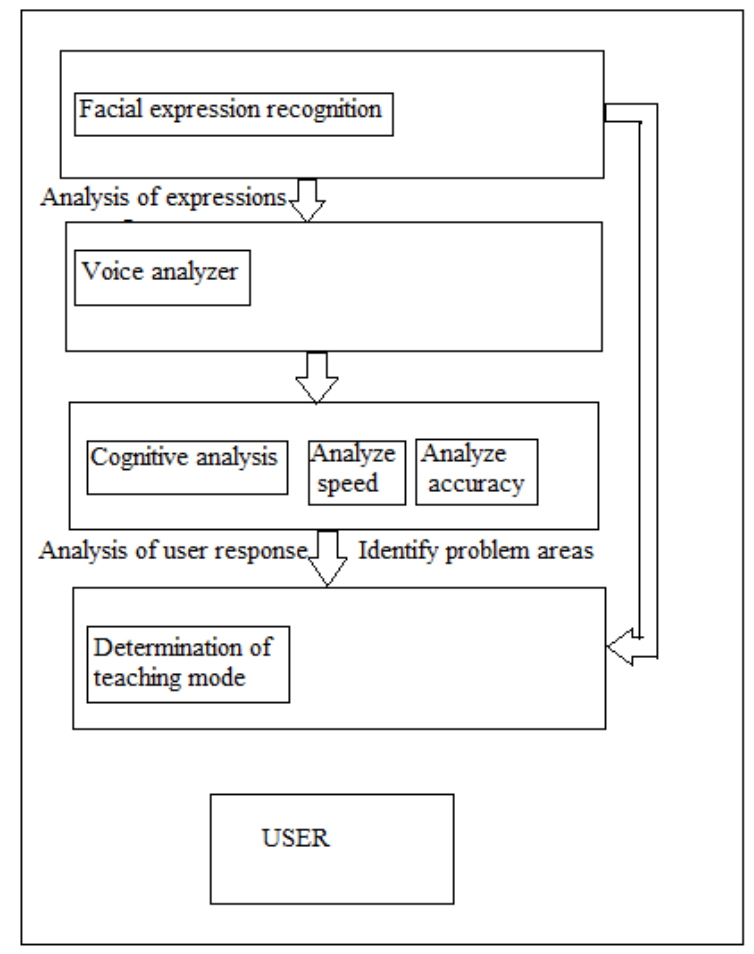

Fig 1: Block diagram of emotionally intelligent tutoring system

\subsection{Facial expression recognition}

Face recognition programs on computers work by measuring prominent features of the face, such as the pupils of the eyes and the tip of the nose. The distances and angles between these are different for every face.

Computers of today are fairly advanced in detecting emotions of humans and can detect basic facial expressions and gestures. By recognizing expressions like a frown, smile, nod, etc. the system will be able to understand the basic mindset of the user during learning. If the user appears to not understand or seems troubled, the mode of the material can be altered, so as to suit his needs.

By observing the facial expressions, typing speed, tone of voice, whether voice shakes or hesitates, the system can recognize the psychological state of the student. By understanding the emotional state of students to some extent the software can adapt to create a comfort zone for them while learning. For students who seem bored, the system will switch to the interactive mode of the same material. The software shall also have various modes for slow, average and advanced learners as well as students who prefer theory or interactive studies. Based on these factors, the modes can be shifted. There can also be a manual override as the computer is still not completely apt for reading emotions.

\subsection{Analysis of user response}

The course will be divided into various subtopics, with a quiz of some sort in each section. Based on the number of correct answers, speed of completion and voice analysis i.e. whether the user hesitates or voice shakes, indicating discomfort or uncertainty of the subject matter, the system will comprehend the cognitive and emotional state of the user.

\subsection{Identify problem areas}

The system can identify common areas and types of mistakes over the span of the course using information from previous tests and clustering them on the basis of similarity of topic or question type. For example some students might have difficulty remembering facts and formulae while others cannot perceive a practical problem. After this analysis, the system should focus more on the weaknesses so as to help the student like a real teacher would. The problem areas should be identified and more emphasis should be laid on them during review.

\subsection{Modes of the system}

The exceptional thing about the proposed system is that it has different modes of learning for slow, average and advanced learners with each mode being subdivided into theoretical and interactive teaching methods, as opposed to a single course material for all users regardless of their ages, experiences and aptitudes.

The user inputs personal details and is started off into one of the modes of theoretical type. If the user seems bored, the content from the next topic onwards is shifted to interactive mode. After each test, the user is analyzed as mentioned in the previous sections. The analysis shows whether the current mode suits the user. If it does not, the user is subjected to a different mode of higher or lower level base on the analysis. The user is also analyzed in interactive and theoretical modes and is shown the mode which seems to suit them the best. As emotional intelligence is not as accurate as we desire, there is a button to choose the different modes manually as well. 
Table 1. Types of modes

\begin{tabular}{|c|c|c|c|}
\hline & Slow & Average & Advanced \\
\hline Theoretical & $\begin{array}{l}\text { For young } \\
\text { or } \\
\text { inexperience } \\
\text { d users who } \\
\text { prefer } \\
\text { theoretical } \\
\text { study }\end{array}$ & $\begin{array}{l}\text { For average } \\
\text { users who } \\
\text { prefer } \\
\text { theoretical } \\
\text { study }\end{array}$ & $\begin{array}{l}\text { For } \\
\text { experienced } \\
\text { or fast } \\
\text { learners who } \\
\text { prefer } \\
\text { theoretical } \\
\text { study }\end{array}$ \\
\hline Interactive & $\begin{array}{l}\text { For young } \\
\text { or } \\
\text { inexperience } \\
\text { d users who } \\
\text { prefer } \\
\text { interactive } \\
\text { study }\end{array}$ & $\begin{array}{l}\text { For average } \\
\text { users who } \\
\text { prefer } \\
\text { interactive } \\
\text { study }\end{array}$ & $\begin{array}{l}\text { For } \\
\text { experienced } \\
\text { or fast } \\
\text { learners who } \\
\text { prefer } \\
\text { interactive } \\
\text { studies }\end{array}$ \\
\hline
\end{tabular}

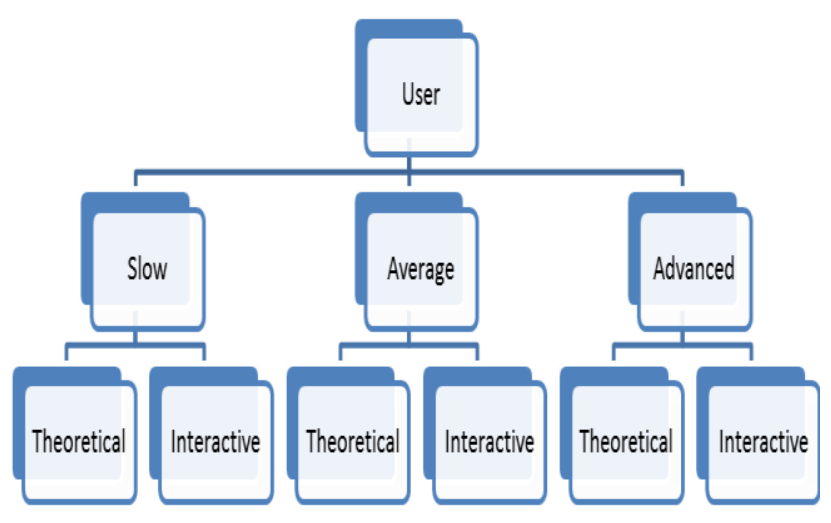

Fig 2: Flowchart depicting modes of the system

\subsection{Feedback system}

Computers are still not perfectly adapted to reading emotions, so a feedback system can be devised to take user input after the course. The user can point out where the system anticipated the emotional states incorrectly, which will help in future development.

\section{FLOWCHART}

The system is depicted using the following flowchart. The mode is decided based on user details.

Slow: For young or inexperienced learner.

Average: For the majority or age appropriate users

Advanced: For older or experienced users

The starting mode can be of any level but is theoretical.

If the user is bored, the system switches to interactive mode. However, another reason for boredom could be that the user is not sufficiently challenged. The system will decide whether to switch to interactive or higher mode by analyzing various factors like facial expression while learning or giving tests, speed of completion, typing speed, voice analysis on giving answers, etc. For a user who seems confused or answers most questions incorrectly the mode is changed to a lower version. If the mode is already the lowest, the type of content is changed i.e. from interactive to theoretical and vice versa and the problem areas are stored for future review.

The tests in between check how much user has understood and retained. After each test, data of incorrect answers and their types are stored.

At the end of the course, the system determines the areas in which the user is weak are reviews them in a different format of the corresponding mode i.e. Review is interactive if the problem topics were covered using theoretical material. This helps the user to review old topics without feeling like he is doing the same thing repeatedly. After the final test, user is asked for feedback to help improve the emotional intelligence of the system 


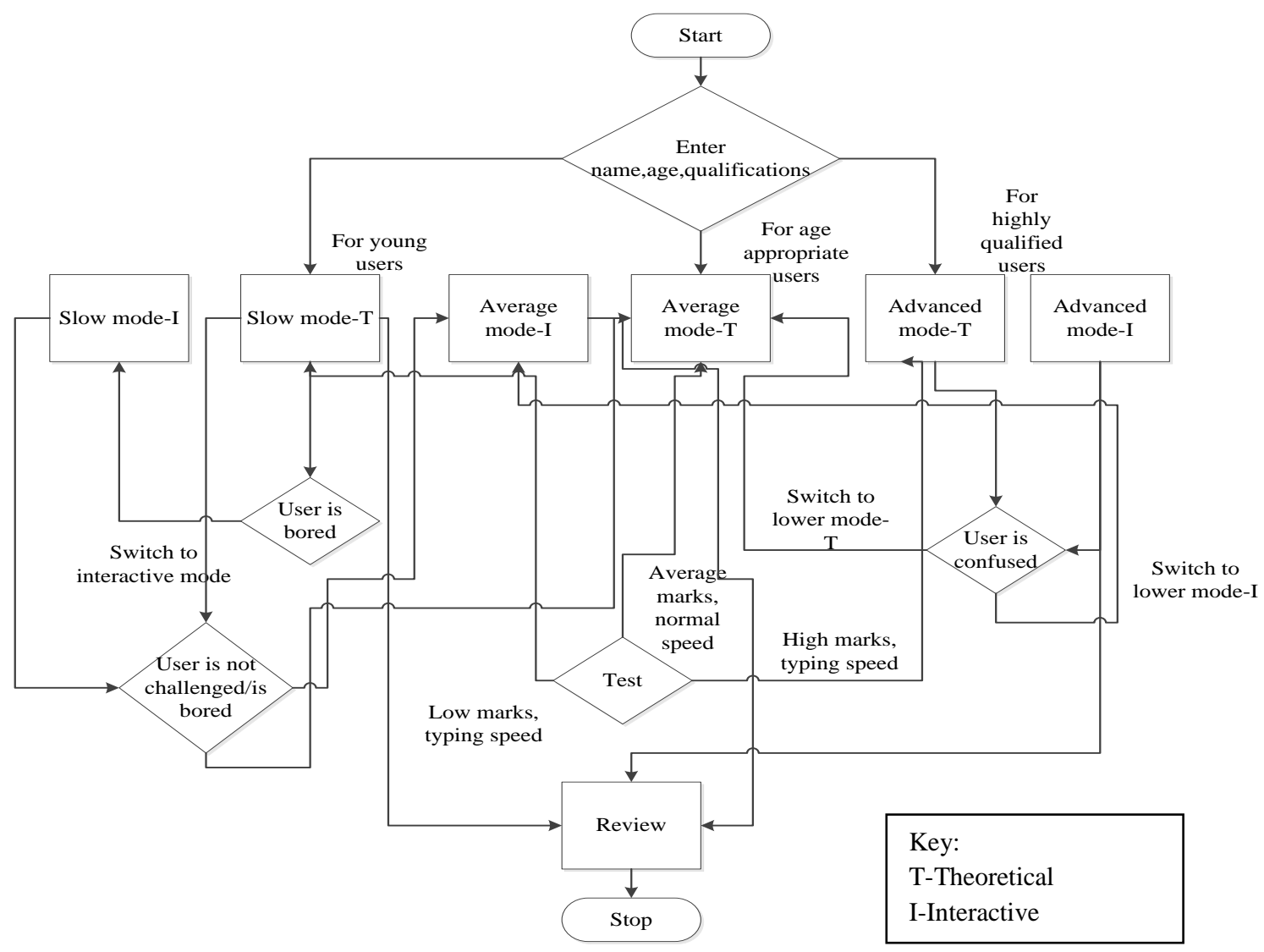

Fig 3: Flowchart depicting aspects of the tutoring system

\section{CONCLUSION}

In this world of technology, with online courses becoming a great way for people to learn and expand their knowledge, it is of immense importance to further develop the content provided by these courses. An emotionally intelligent tutoring system will help pave the way for a better online learning experience by individualizing the course content to a certain extent in order to cater the different needs of the users. Using facial expression and voice recognition software, the proposed system will be able to accurately assess the aptitude and emotions of the users and alter the content accordingly. Analysis of tests will further help determine how much the user has learnt and where he or she faces a difficulty. Thus, such a tutoring agent will improve the learning experience tremendously and reduce the number of dropouts from online courses as it will not leave room for boredom for advanced users nor provide material that goes over the head of other users.

This paper demonstrates that with the integration of emotional intelligence in MOOCs, online learning and distance education will be able to progress to new heights. Thus, the proposed system will help improve the existing quality and content of online tutoring courses. As opposed to a single source of material of all types of users, the proposed system will cater to a plethora of different users and create a unique learning experience that will be correct for each individual.

\section{ACKNOWLEDGMENTS}

I would like to thank VIT University for teaching me and inculcating the importance of research in me.

\section{REFERENCES}

[1] Department of Psychology, University of Toronto "Can Computers Possess Emotional Intelligence", http://psych.utoronto.ca/users/reingold/courses/ai/emotio nal.html

[2] Motherboard, "Emotionally Intelligent Machines Are Closer Than Ever" $\mathrm{http}: / /$ motherboard.vice.com/blog/emotionallyintelligent-machines-are-closer-than-ever

[3] University of Memphis, Institute for Intelligent Systems, http://en.wikipedia.org/wiki/AutoTutor

[4] Auto Tutor, http://www.autotutor.org/

[5] Facial expression analysis, http://www.affdex.com

[6] Affectiva, http://en.wikipedia.org/wiki/Affectiva.

[7] Beyond Verbal, http://www.beyondverbal.com/

[8] Amir Mizroch, "App Tells You How You Feel", The Wall Street Journal

[9] Christine L. Lisetti, Diane J. Schiano, “Automatic Facial Expression Interpretation: Where Human-Computer Interaction, Artificial Intelligence and Cognitive Science Intersect", Pragmatics and Cognition (Special Issue on Facial Information Processing: A Multidisciplinary Perspective), Vol. 8(1): 185-235, 2000.

[10] Dexter Johnson, "MOOCs Teach AI to Grade Computer Programs as a Human Would", http://spectrum.ieee.org/at-work/education/moocs-teachai-to-grade-computer-programs-as-a-human-would 